\title{
Mechanisms of Resistance to CDK4/6 Inhibitors in Breast Cancer and Potential Biomarkers of Response
}

\author{
Cristina Guarduccia Martina Bonechi ${ }^{a}$ Giulia Boccalini ${ }^{a} \quad$ Matteo Benellib Emanuela Risi ${ }^{b}$ \\ Angelo Di Leo ${ }^{b}$ Luca Malorni ${ }^{a, b} \quad$ Ilenia Migliaccio ${ }^{a}$
}

a 'Sandro Pitigliani' Medical Oncology Department, Hospital of Prato, Istituto Toscano Tumori, Prato, Italy;

b'Sandro Pitigliani' Medical Oncology Department, Hospital of Prato, Istituto Toscano Tumori, Prato, Italy

\section{Keywords \\ CDK4/6 inhibitors - Drug resistance - Breast cancer}

\section{Summary}

Randomized clinical trials demonstrated that CDK4/6 inhibitors are highly effective in patients with hormone receptor-positive (HR+), HER2-negative (HER2-) metastatic breast cancer in combination with endocrine therapy. The use of CDK4/6 inhibitors in clinics is becoming common for patients with HR+/HER2- metastatic breast cancer and will certainly increase in the near future. However, patients might show de novo or acquired resistance to these drugs. Molecular alterations have been suggested as determinants for de novo resistance to CDK4/6 inhibitors, but have never been validated in a clinical setting. In addition, molecular mechanisms of acquired resistance to palbociclib have been analyzed only in preclinical studies. Here we review the current knowledge on the available preclinical data about the mechanisms of de novo and acquired resistance to CDK4/6 inhibitors in breast cancer, and clinical data about potential biomarkers of response.

(C) 2017 S. Karger GmbH, Freiburg

\section{CDK4/6 Pathway}

In most adult tissues, differentiated cells are maintained in a quiescent state (G0 phase). Mitogenic stimuli, such as growth factors and hormones, can trigger the cell cycle inducing the progression from G0 or Gap1 (G1) phases to synthesis (S) phase.
The progression from G1 to S phase is a particularly critical checkpoint, a key effector of this checkpoint being the retinoblastoma susceptibility gene product $(\mathrm{Rb})[1]$. $\mathrm{Rb}$ is an onco-suppressor whose primary function, as for other Rb family members (such as p107 and p130), is to repress the transcription of genes required for the S-phase entry, preventing the unscheduled progression through the cell cycle. In particular, Rb inhibits the transcription factors of the E2F family, which regulate genes involved in cell cycle control, mitotic progression and dNTP biosynthesis $[1,2]$. During cell cycle, $\mathrm{Rb}$ activity is regulated through phosphorylation by the cyclin-dependent kinases (CDK), a group of serine/threonine kinases whose activity depends on binding of regulatory proteins named cyclins [3]. The G1/S transition is promoted by Dtype cyclins (D1, D2 and D3) and the CDK4 and CDK6, the initial CDKs to phosphorylate $\mathrm{Rb}$, which is then phosphorylated by other complexes such as cyclin E/CDK2 [4]. Phosphorylation leads to $\mathrm{Rb}$ functional inactivation and the consequent release of E2Fs, which activate the transcription of genes triggering the S-phase entry $[1,2]$.

CDK activity and progression of the cell cycle through the G1/S checkpoint are regulated by the CDK inhibitors of the Cip-Kip family, including p21 and p27, and specific CDK4 and CDK6 inhibitors of the INK4 family, such as p16 [5].

\section{CDK4/6 Inhibitors}

Palbociclib (PD0332991), abemaciclib (LY2835219) and ribociclib (LEE011) are selective ATP-competitive, orally administered inhibitors of CDK4 and CDK6. These compounds have been extensively studied in vitro and in vivo and are currently in different stages of clinical development [3].

Palbociclib is a highly specific inhibitor of CDK4 (half maximal inhibitory concentration (IC50), $11 \mathrm{nM}$ ) and CDK6 (IC50, $16 \mathrm{nM}$ ),

\section{KARGER}

() 2017 S. Karger GmbH, Freiburg 
and was the first of these compounds to be synthesized and tested in clinical trials $[6,7]$. Palbociclib has received approval by the US Food and Drug Administration (FDA) and by the European Medicines Agency (EMA) for the treatment of hormone receptor-positive HER2-negative (HR+/HER2-) advanced breast cancer in combination with the hormonal treatments letrozole or fulvestrant $[8$, 9], given the unprecedented results of three pivotal randomized clinical trials, the PALOMA-1, PALOMA-2 and PALOMA-3 [1012]. PALOMA-1 and PALOMA-2 are phase II and phase III randomized trials, respectively, of palbociclib in combination with letrozole versus letrozole alone for previously untreated patients in the metastatic setting $[10,11]$. PALOMA-3 is a phase III randomized trial of palbociclib and fulvestrant versus placebo and fulvestrant for the treatment of patients progressing on a previous hormonal therapy [12]. These trials demonstrated the superiority of the combination over the hormonal treatment alone in both untreated and in hormone pre-treaded populations [10-12].

Ribociclib was the second specific CDK4/6 inhibitor to receive FDA approval in combination with hormonal therapy for the firstline treatment of postmenopausal women with metastatic $\mathrm{HR}+$ / HER2- breast cancer [13]. The approval was based on the results of the phase III MONALEESA-2 trial showing a significantly longer progression-free survival (PFS) in the ribociclib group than in the placebo group [14].

Abemaciclib inhibits CDK4/cyclin D1 and CDK6/cyclin D1 with an IC50 of $2 \mathrm{nM}$ and $10 \mathrm{nM}$, respectively [15]. Abemaciclib has been granted by the FDA breakthrough therapy designation for patients with refractory HR+ metastatic breast cancer based on the encouraging results from a phase I study in which single-agent abemaciclib demonstrated a clinical benefit rate (CBR) of $61 \%$ in patients with heavily pretreated metastatic breast cancer [16].

Despite the tolerability shown by palbociclib, abemaciclib and ribociclib, these drugs are not devoid of side effects, with neutropenia being a common adverse event $[10,11,14,16,17]$. Therefore, two next-generation CDK4/6 inhibitors with lower myelosuppression activity, G1T28 and G1T38, have been recently developed and are currently being tested in phase I/II clinical trials [18, 19].

Given the efficacy shown by CDK4/6 inhibitors, the clinical use of these drugs is becoming common for patients with HR+/HER2advanced breast cancer, and will certainly increase in the near future. Notwithstanding, results from clinical trials indicate that patients might show de novo resistance to CDK4/6 inhibitors, and that even initially responding patients will eventually acquire resistance to these drugs $[10-12,14,20]$. Although preclinical studies have suggested biological determinants of de novo resistance, to date no biomarker has been clinically validated in patients with HR+/HER2- breast cancer. In addition, there is limited knowledge, even preclinically, on the mechanisms of acquired resistance to palbociclib.

Here we review preclinical data about the mechanisms of de novo and acquired resistance to CDK4/6 inhibitors in breast cancer and clinical data on potential biomarkers of response. Published literature has been reviewed up to April 2017.

\section{Mechanisms of Resistance}

\section{De Novo Resistance}

$\mathrm{Rb}$ is the main target of CDK4/6, representing a key mediator of CDK4/6 inhibition. The vast majority of preclinical studies suggests that $\mathrm{Rb}$ loss confers resistance to CDK4/6 inhibitors. Indeed, CDK4/6 inhibitors are able to effectively block proliferation of a variety of human $\mathrm{Rb}$-positive tumors, including breast cancer, both in vitro and in vivo, while Rb-negative tumor cells are largely resistant to CDK4/6 inhibitors [6, 7, 21-25]. In a pivotal study investigating the in vitro effects of palbociclib in a panel of 47 human breast cancer cell lines, RB1, CCND1 (cyclin D1) and CDKN2A (p16) were differentially expressed between sensitive and de novo resistant cells. In particular, higher levels of $R B 1$ and $C C N D 1$, and lower levels of CDKN2A were found in the sensitive group [25]. In addition, for the first time, a statistically significant correlation between molecular subtypes and sensitivity to palbociclib was shown, with the most sensitive subtypes being those that were ER+, including the HER2-amplified [25]. The role of Rb and p16 in determining resistance/sensitivity to palbociclib has been also confirmed in an ex vivo model of breast tumor tissue in which tumor explants were cultured and treated with palbociclib. In this model system, response was not dependent on ER or HER2 status, but tumors with high levels of p16 or loss of Rb failed to respond to palbociclib [23]. In line with the key role of the Rb pathway in determining de novo resistance to palbociclib, we have recently shown that a gene expression signature of retinoblastoma loss-of-function, the RBsig, and a previously developed signature of retinoblastoma loss of heterozygosity (LOH) [26] were able to discriminate palbociclib resistant and sensitive breast cancer cell lines [27].

Besides deregulation of $\mathrm{Rb}$, other mechanisms are likely to be implicated in the de novo resistance to CDK4/6 inhibitors. Indeed, in the study of Finn et al. [25], 3 cell lines with detectable levels of $\mathrm{Rb}$ were still resistant and did not show a decrease in Rb phosphorylation after palbociclib treatment, suggesting that $\mathrm{Rb}$ status is not the only determinant of resistance to palbociclib. In addition, it has been shown that, after induction of loss of $\mathrm{Rb}$ in 3 different cell lines, cells still responded to palbociclib treatment, and proliferation was significantly reduced irrespective of $\mathrm{Rb}$ status, suggesting that $\mathrm{Rb}$ protein is not necessarily required for the response to CDK4/6 inhibition [24]. On the other hand, overexpression of E2F2 was able to promote the bypass of CDK4/6 inhibition, more than loss of $\mathrm{Rb}$ alone, implying an incomplete relationship between the cyclin D/CDK4/6/RB axis and E2F regulation [24]. Also, palbociclib was shown to be less effective at reducing proliferation of cyclin E1- or cyclin E2-overexpressing breast cancer cells compared to vector control cells, and resistance to palbociclib was no longer evident when combination of CDK2 and CDK4 inhibitors was used, suggesting that cyclin E/CDK2 complexes might mediate resistance to CDK4/6 inhibitors [28].

Other suggested mechanisms of de novo resistance to CDK4/6 inhibitors, involving deregulation of cell cycle-related proteins, include phosphorylation status of p27 and fizzy-related protein homolog (FZR1) $[29,30]$. In addition to its ability to inhibit cyclin D/ 
CDK4, p27 can regulate the cyclin D/CDK4/p27 ternary complex activity depending on phosphorylation status of tyrosine Y74, Y88, and Y89, [31]. It was shown that overexpression of Brk (breast tumor-related kinase), an intracellular tyrosine kinase overexpressed in $60 \%$ of breast cancers [32], increases phosphorylation on Y88 of p27 and cyclin D/CDK4 activity, rendering breast cancer cells more resistant to palbociclib [29].

FZR1 functions as a specific activator of APC/C (anaphase-promoting complex or cyclosome) ubiquitin ligase, regulating late mitosis and G1/S phase [33]. It was shown that in human breast cancer cells simultaneous knockdown of Rb and FZR1 synergistically bypassed cell division arrest induced by palbociclib, suggesting that not only Rb status but also FZR1 levels and APC/C FZR1 functional activity might determine how cancer cells respond to CDK4/6 inhibitors [30].

In breast cancer, ESR1 mutations have been associated with resistance to aromatase inhibitors [34]. One question might be whether cells with ESR1 mutations respond to CDK4/6 inhibitors. It was shown that the expression of the Y537S, Y537N, or D538G ESR1 mutations in T47D breast cancer cells did not negatively impact the efficacy of the combination of palbociclib with a selective estrogen receptor (ER) down-regulator [35]. Also, in 1 patient-derived xenograft (PDX) model harboring the D538G ESR mutation and an activating PIK3CA mutation, significant efficacy was observed with palbociclib alone or in combination with fulvestrant. However, longterm delayed tumor growth was only observed when palbociclib was co-administered with fulvestrant [36]. In addition, palbociclib alone did not induce tumor regression in an MCF7 model harboring an Y537S mutation in 1 ER allele and a frame-shift mutation in another ER allele [36]. The role of palbociclib alone in breast cancer cells harboring ESR1 mutation needs to be further clarified.

\section{Acquired Resistance}

To date, few studies have investigated the mechanisms of acquired resistance to CDK4/6 inhibitors in breast cancer. As in de novo resistance, loss of $\mathrm{Rb}$ has been implicated in acquired resistance to palbociclib. Indeed, loss of Rb expression was detected in an in vitro model of palbociclib-resistant cell lines and a PDX model of acquired resistance to ribociclib showed a sub-clonal selection of an $R B 1$ frameshift mutation [37].

However, the majority of data suggests that deregulation of cyclin $\mathrm{E} / \mathrm{CDK} 2$ or $\mathrm{PI} 3 \mathrm{~K} / \mathrm{AKT} / \mathrm{mTOR}$ signaling might contribute to a therapeutic bypass to CDK4/6 inhibitors. It has been demonstrated that, following CDK4/6 inhibition, breast cancer cells show adaptation as early as $72 \mathrm{~h}$ [37]. This was shown to be mediated by noncanonical activation of cyclin D1/CDK2 complexes, which in turn induced a recovery of cyclin E2 expression and S-phase entry despite CDK4/6 inhibition. PI3K inhibition was able to reduce cyclin D1 expression and prevent early adaptation [37]; also, the combination of PI3K and CDK4/6 inhibitors has shown substantial antitumor activity in vivo $[37,38]$, suggesting a potential therapeutic strategy to prevent early adaptation to palbociclib.

Amplification of CCNE1 was found in MCF7 with acquired resistance to palbociclib. Silencing of CCNE1 or CDK2 resulted in substantially increased cell-cycle arrest and reduction in cell growth in combination with palbociclib even though in this model PI3K inhibition was unable to re-sensitize cells to palbociclib [37]. Dean et al. [24], analyzing MCF10A (a non-tumorigenic cell line) and the ER-negative MDAMB231 cell lines that were able to grow after extended palbociclib treatment, similarly suggested that deregulation of CDK2 and loss of CDK inhibitors p21 and p27, which function on CDK2, could represent a mechanism leading to bypass of palbociclib. The 3-phosphoinositide-dependent protein kinase 1 (PDK1), which functions downstream of PI3K and is required for the full activation of AKT, was found to be a key factor for ribociclib response in vitro [39]. PDK1 was induced by CDK4/6 inhibitors in vitro and its expression was sustained in ribociclib-resistant cell lines together with increased levels of pAKT at S477/T479, a CDK2-dependent phosphorylation site. Genetic and pharmacological inhibition of PDK1, CDK2 or PI3K/AKT/mTOR inhibition in combination with ribociclib re-sensitized cells to ribociclib, suggesting that enhanced PDK1 expression and PI3K/PDK1/AKT/ mTOR signaling mediate acquired resistance to CDK4/6 inhibition [39].

Finally, while a model of CAMA1 with acquired resistance to abemaciclib was found to have overexpression of CCNE1, similar to that seen previously with palbociclib [40], a model of abemaciclib-resistant MCF7 showed an increase in CDK6 mRNA levels with concomitant increase in CDK6 protein levels. In addition, a fraction of the resistant cells showed an increased CDK6 copy number. Reducing CDK6 levels restored the sensitivity, while overexpression of CDK6 resulted in reduced sensitivity to abemaciclib [40], suggesting that overexpression/amplification of CDK6 might sustain abemaciclib resistance. In addition, both models showed reduced expression of ESR1 and PGR [40], which was associated with reduced sensitivity to hormonal treatments in MCF7-resistant cells [40]. Intriguingly, on a limited number of tumor biopsies from patients treated with abemaciclib or ribociclib, it appeared that tumors changed from ER+ to ER- or from PR+ to PR- [40], supporting the preclinical data suggesting that ER might be implicated in CDK4/6 inhibitors resistance.

\section{Potential Biomarkers}

Based on preclinical data suggesting Rb, p16 and cyclin D1 as determinants of response to CDK4/6 inhibitors, some early trials with palbociclib either selected or analyzed patients based on these biomarkers. However, these trials do not seem to support the use of these biomarkers for selecting patients for CDK4/6 inhibitors treatment.

A phase II, single-arm trial of palbociclib enrolled 37 patients with advanced breast cancer whose tumors were $\mathrm{Rb}$ positive [41]. $\mathrm{Rb}$ expression/localization, the proliferation index Ki-67, p16 loss and CCND1 amplification were assessed on archival tumor tissue from either breast primary tumors or metastatic lesions to identify subgroups of patients with different sensitivity to palbociclib [41]. None of the biomarkers analyzed was significantly associated with 
either CBR or PFS in the overall population or in the HR+ subgroup [41]. Additionally, in this population specifically selected to have $\mathrm{Rb}$-positive tumors, CBR was low (19\% and $21 \%$ overall and in the HR+ cohort, respectively), although patients were heavily pretreated [41]. Interestingly, CBR was slightly higher (29\%) among patients who had progressed through at least 2 prior lines of hormonal therapy, suggesting activity in the setting of acquired endocrine resistance [41]. Of note, 2 patients with HR+/HER2positive (HER2+) tumors responded [41] in line with preclinical data on HER2+ tumors [25].

The PALOMA 1 trial enrolled patients in 2 different cohorts [10]. In cohort 2, patients were required to have cancers with amplification of CCND1, loss of p16, or both [10]. However, after an interim analysis of cohort 1 that showed clinically meaningful activity of the combination, enrolment into cohort 2 was stopped because patient selection based on CCND1 amplification or p16 loss was deemed unlikely to further improve patient outcome [10]. Indeed, results of the trial did not seem to support the hypothesis that this biomarker selection could discriminate responsive versus nonresponsive tumors [10]. In addition, in an extended analysis of this trial, palbociclib plus letrozole improved median PFS and the CBR regardless of age, histological type, prior neoadjuvant/adjuvant systemic treatment and disease site [17].

In the PALOMA 3 trial, the effects on treatment of endocrine therapy resistance, quantitative HR expression, and tumor PIK3CA or ESR1 mutational status were analyzed [20,42]. The efficacy of fulvestrant plus palbociclib was not affected by the number of previous endocrine therapies, reported sensitivity to previously received endocrine therapy, level of expression of ER and PR, or PIK3CA mutational status analyzed at baseline in circulating DNA [20]. Also, fulvestrant plus palbociclib improved PFS in both ESR1 mutant and ESR1 wild-type patients [42], suggesting that these markers might not distinguish sensitive/resistant patients. Interestingly, in a study analyzing 16 patients treated with palbociclib and letrozole, it was shown that the combination did not prevent the selection of ESR1 mutations. Indeed, longitudinal tracking of ESR1 mutations in serial blood draws revealed selection of mutations in 3 out of 4 patients while on palbociclib and letrozole therapy [43].

The neoadjuvant phase II trial NeoPalAna was designed to determine whether anastrozole and palbociclib induce a higher rate of complete cell cycle arrest (CCCA: Ki-67 < 2.7\%) compared to anastrozole alone in patients with stage II/III ER+/HER2- newly diagnosed breast cancer [44]. The study was also aimed at discovering potential predictive biomarkers [44]. Tumor biopsies were collected at baseline (C0D1), at C1D1 following 4 weeks of anastrozole monotherapy, and at C1D15, 2 weeks after the addition of palbociclib to anastrozole; tumor specimens from surgery were also available [44]. The rates of CCCA with palbociclib plus anastrozole were significantly higher at C1D15 than at C1D1 with anastrozole monotherapy [44]. As expected, in this study the 2 non-luminal tumors were resistant, but Ki-67 levels were significantly reduced by palbociclib in both luminal A and luminal B tumors [44], suggesting activity irrespectively of luminal subtypes. Palbociclib benefit was observed independently of grade, negativity for PR, or muta- tions in TP53, PTEN or PIK3CA [44]. Intriguingly, RB1 mutation was identified in 3 breast cancers, 2 of which were still sensitive to palbociclib [44], in line with the hypothesis that additional factors besides $\mathrm{Rb}$ might be implicated in resistance to CDK4/6 inhibitors. Gene expression data showed elevated expression of CCND3, CCNE1 and CDKN2D at C1D15 in resistant tumors, suggesting a persistent E2F activity in non-responding tumors [44].

Biomarkers of resistance/response in breast cancer patients are also lacking for ribociclib and abemaciclib. In the phase III trial of ribociclib, the combination of ribociclib and letrozole improved PFS over letrozole and placebo regardless of age, HR status, site of metastatic disease and previous therapy [14]. In the multicenter study in which single-agent abemaciclib was administered to a cohort of 47 breast cancer patients, responses were not limited to patients with HR+ HER2- disease and also included those with HR+ HER2 + breast cancer [16]. In line with palbociclib data, abemaciclib demonstrated clinical activity in $\mathrm{HR}+$ breast cancers irrespective of PIK3CA mutational status [16].

ER positivity and HER2 negativity are currently the only markers used to select breast cancer patients for CDK4/6 inhibitors treatment in clinics.

\section{Conclusions}

CDK4/6 inhibitors represent a new, effective, therapeutic option for patients with metastatic luminal breast cancer. Despite preclinical evidence suggesting that numerous mechanisms can contribute to de novo or acquired resistance to these agents, there is as yet no biomarker that can be used clinically to rationally allocate patients to these compounds. It is likely that, given the complexity of the biology of the CDK4/6 pathway, multiple mechanisms of resistance to CDK4/6 inhibitors might operate in different patients, even concomitantly, therefore making biomarker research in this field particularly challenging. Given the clinical relevance of this topic, further research is needed to personalize treatment strategies in this setting.

\section{Acknowledgements}

The authors would like to thank AIRC (Associazione Italiana per la Ricerca sul Cancro) and Fondazione 'Sandro Pitigliani' per la lotta contro i tumori ONLUS for their generous support.

\section{Disclosure Statement}

L.M.: Lecture, Advisory board from AstraZeneca and Pfizer; Research support from Pfizer. A.D.L.: Consulting Fees (e.g. advisory boards) from DaichiiSankyo, Roche, Novartis, Pfizer, AstraZeneca, Genomic Health, Eisai, Lilly, Pierre Fabre, Bayer, Celgene, Puma Biotechnology; Contracted Research from Novartis, Pfizer, AstraZeneca; Other (Travel funds, Honoria) from AstraZeneca, Bayer, Celgene, Daichii-Sankyo, Eisai, Genomic Health, Ipsen, Lilly, Novartis, Pfizer, Pierre Fabre, Roche. C.G., M.B., G.B., M.B., E.R. and I.M.: No conflict of interest. 


\section{References}

1 Henley SA, Dick FA: The retinoblastoma family of proteins and their regulatory functions in the mammalian cell division cycle. Cell Div 2012;7:10.

$\checkmark 2$ Giacinti C, Giordano A: RB and cell cycle progression. Oncogene 2006;25:5220-5227.

3 Asghar U, Witkiewicz AK, Turner NC, Knudsen ES: The history and future of targeting cyclin-dependent kinases in cancer therapy. Nat Rev Drug Discov 2015; 14:130-146.

4 Musgrove EA, Caldon CE, Barraclough J, et al.: Cyclin $\mathrm{D}$ as a therapeutic target in cancer. Nat Rev Cancer 2011;11:558-572.

5 Sherr CJ, Roberts JM: Inhibitors of mammalian G1 cyclin-dependent kinases. Genes Dev 1995;9:1149-1163.

6 Fry DW, Harvey PJ, Keller PR, et al.: Specific inhibition of cyclin-dependent kinase 4/6 by PD 0332991 and associated antitumor activity in human tumor xenografts. Mol Cancer Ther 2004;3:1427-1438.

7 Toogood PL: Cyclin-dependent kinase inhibitors for treating cancer. Med Res Rev 2001;21:487-498.

8 Beaver JA, Amiri-Kordestani L, Charlab R, et al.: FDA approval: Palbociclib for the treatment of postmenopausal patients with estrogen receptor-positive, HER2negative metastatic breast cancer. Clin Cancer Res 2015;21:4760-4766.

9 Walker AJ, Wedam S, Amiri-Kordestani L, et al.: FDA approval of palbociclib in combination with fulvestrant for the treatment of hormone receptor-positive, HER2-negative metastatic breast cancer. Clin Cancer Res 2016;22:4968-4972.

10 Finn RS, Crown JP, Lang I, et al.: The cyclin-dependent kinase 4/6 inhibitor palbociclib in combination with letrozole versus letrozole alone as first-line treatment of oestrogen receptor-positive, HER2-negative, advanced breast cancer (PALOMA-1/TRIO-18): a randomised phase 2 study. Lancet Oncol 2015;16:25-35.

-11 Finn RS, Martin M, Rugo HS, et al.: Palbociclib and letrozole in advanced breast cancer. N Engl J Med 2016;375:1925-1936.

12 Turner NC, Ro J, Andre F, et al.: Palbociclib in hormone-receptor-positive advanced breast cancer. N Engl J Med 2015;373:209-219.

13 Ribociclib approved for advanced breast cancer. Cancer Discov 2017;7:OF3.

14 Hortobagyi GN, Stemmer SM, Burris HA, et al.: Ribociclib as first-line therapy for HR-positive, advanced breast cancer. N Engl J Med 2016;375:1738-1748.

15 Gelbert LM, Cai S, Lin X, et al.: Preclinical characterization of the CDK4/6 inhibitor LY2835219: in-vivo cell cycle-dependent/independent anti-tumor activities alone/in combination with gemcitabine. Invest New Drugs 2014;32:825-837.

16 Patnaik A, Rosen LS, Tolaney SM, et al.: Efficacy and safety of abemaciclib, an inhibitor of CDK4 and CDK6, for patients with breast cancer, non-small cell lung cancer, and other solid tumors. Cancer Discov 2016;6:740-753.

17 Finn RS, Crown JP, Ettl J, et al.: Efficacy and safety of palbociclib in combination with letrozole as first-line treatment of ER-positive, HER2-negative, advanced breast cancer: expanded analyses of subgroups from the randomized pivotal trial PALOMA-1/TRIO-18. Breast Cancer Res 2016;18:67.
Bisi JE, Sorrentino JA, Jordan JL, et al.: Preclinical development of G1T38: a novel, potent and selective inhibitor of cyclin dependent kinases $4 / 6$ for use as an oral antineoplastic in patients with CDK4/6 sensitive tumors. Oncotarget 2017;8:42343-42358.

19 Bisi JE, Sorrentino JA, Roberts PJ, et al.: Preclinical characterization of G1T28: a novel CDK4/6 inhibitor for reduction of chemotherapy-induced myelosuppression. Mol Cancer Ther 2016;15:783-793.

20 Cristofanilli M, Turner NC, Bondarenko I, et al.: Fulvestrant plus palbociclib versus fulvestrant plus placebo for treatment of hormone-receptor-positive, HER2-negative metastatic breast cancer that progressed on previous endocrine therapy (PALOMA-3): final analysis of the multicentre, double-blind, phase 3 randomised controlled trial. Lancet Oncol 2016;17:425-439.

21 Young RJ, Waldeck K, Martin C, et al.: Loss of CD$\mathrm{KN} 2 \mathrm{~A}$ expression is a frequent event in primary invasive melanoma and correlates with sensitivity to the CDK4/6 inhibitor PD0332991 in melanoma cell lines. Pigment Cell Melanoma Res 2014;27:590-600.

22 Zhang YX, Sicinska E, Czaplinski JT, et al.: Antiproliferative effects of CDK4/6 inhibition in CDK4-amplified human liposarcoma in vitro and in vivo. Mol Cancer Ther 2014;13:2184-2193.

23 Dean JL, McClendon AK, Hickey TE, et al.: Therapeutic response to CDK4/6 inhibition in breast cancer defined by ex vivo analyses of human tumors. Cell Cycle 2012;11:2756-2761.

24 Dean JL, Thangavel C, McClendon AK, et al.: Therapeutic CDK4/6 inhibition in breast cancer: key mechanisms of response and failure. Oncogene 2010;29: 4018-4032.

25 Finn RS, Dering J, Conklin D, et al.: PD 0332991, a selective cyclin D kinase 4/6 inhibitor, preferentially inhibits proliferation of luminal estrogen receptor-positive human breast cancer cell lines in vitro. Breast Cancer Res 2009;11:R77.

26 Herschkowitz JI, He X, Fan C, Perou CM: The functional loss of the retinoblastoma tumour suppressor is a common event in basal-like and luminal B breast carcinomas. Breast Cancer Res 2008;10:R75.

27 Malorni L, Piazza S, Ciani Y, et al.: A gene expression signature of retinoblastoma loss-of-function is a predictive biomarker of resistance to palbociclib in breast cancer cell lines and is prognostic in patients with ER positive early breast cancer. Oncotarget 2016;7:6801268022.

28 Caldon CE, Sergio CM, Kang J, et al.: Cyclin E2 overexpression is associated with endocrine resistance but not insensitivity to CDK2 inhibition in human breast cancer cells. Mol Cancer Ther 2012;11:1488-1499.

29 Patel P, Asbach B, Shteyn E, et al.: Brk/protein tyrosine kinase 6 phosphorylates $\mathrm{p} 27 \mathrm{KIP} 1$, regulating the activity of cyclin D-cyclin-dependent kinase 4 . Mol Cell Biol 2015;35:1506-1522.
30 The I, Ruijtenberg S, Bouchet BP, et al.: Rb and FZR1/ Cdh1 determine CDK4/6-cyclin D requirement in C. elegans and human cancer cells. Nat Commun 2015;6: 5906.

31 Blain SW: Switching cyclin D-Cdk4 kinase activity on and off. Cell Cycle 2008;7:892-898.

32 Goel RK, Lukong KE: Tracing the footprints of the breast cancer oncogene BRK - past till present. Biochim Biophys Acta 2015;1856:39-54.

33 Ramanujan A, Tiwari S: APC/C and retinoblastoma interaction: cross-talk of retinoblastoma protein with the ubiquitin proteasome pathway. Biosci Rep 2016; 36:pii: e00377.

34 Jeselsohn R, Buchwalter G, De Angelis C, et al.: ESR1 mutations - a mechanism for acquired endocrine resistance in breast cancer. Nat Rev Clin Oncol 2015;12: 573-583.

35 Wardell SE, Ellis MJ, Alley HM, et al.: Efficacy of SERD/SERM hybrid-CDK4/6 inhibitor combinations in models of endocrine therapy-resistant breast cancer. Clin Cancer Res 2015;21:5121-5130.

36 Ladd B, Mazzola AM, Bihani T, et al.: Effective combination therapies in preclinical endocrine resistant breast cancer models harboring ER mutations. Oncotarget 2016;7:54120-54136.

37 Herrera-Abreu MT, Palafox M, Asghar U, et al.: Early adaptation and acquired resistance to CDK4/6 inhibition in estrogen receptor-positive breast cancer. Cancer Res 2016;76:2301-2313.

38 Vora SR, Juric D, Kim N, et al.: CDK 4/6 inhibitors sensitize PIK3CA mutant breast cancer to PI3K inhibitors. Cancer Cell 2014;26:136-149.

39 Jansen VM, Bhola NE, Bauer JA, et al.: Kinome-wide RNA interference screen reveals a role for PDK1 in acquired resistance to CDK4/6 inhibition in ER-positive breast cancer. Cancer Res 2017;77:2488-2499.

40 Yang C, Li Z, Bhatt T, et al.: Acquired CDK6 amplification promotes breast cancer resistance to CDK4/6 inhibitors and loss of ER signaling and dependence. Oncogene 2017;36:2255-2264.

41 DeMichele A, Clark AS, Tan KS, et al.: CDK 4/6 inhibitor palbociclib (PD0332991) in Rb+ advanced breast cancer: phase II activity, safety, and predictive biomarker assessment. Clin Cancer Res 2015;21:995-1001.

42 Fribbens C, O'Leary B, Kilburn L, et al.: Plasma ESR1 mutations and the treatment of estrogen receptor-positive advanced breast cancer. J Clin Oncol 2016;34: 2961-2968.

43 Gyanchandani R, Kota KJ, Jonnalagadda AR, et al.: Detection of ESR1 mutations in circulating cell-free DNA from patients with metastatic breast cancer treated with palbociclib and letrozole. Oncotarget 2016;8: 66901-66911.

44 Ma CX, Gao F, Luo J, et al.: NeoPalAna: Neoadjuvant palbociclib, a cyclin-dependent kinase 4/6 inhibitor, and anastrozole for clinical stage 2 or 3 estrogen receptor positive breast cancer. Clin Cancer Res 2017;23: 4055-4065. 